\title{
Bridge pier scour measurement by means of Bragg grating arrays
}

\author{
F. Ballio ${ }^{1}$, A. Cigada ${ }^{2}$, G. Crotti ${ }^{1}$, F. Inzoli ${ }^{3}$, and S. Manzoni ${ }^{2, a}$ \\ ${ }^{1}$ Politecnico di Milano, D.I.I.A.R. - Piazza Leonardo da Vinci 32, 20133 Milan (Italy) \\ ${ }^{2}$ Politecnico di Milano, Department of Mechanics - Via La Masa 1, 20156 Milan (Italy) \\ ${ }^{3}$ Politecnico di Milano, Department of Energy - Via Lambruschini 4, 20156 Milan (Italy)
}

\begin{abstract}
This paper deals with a new method to measure scour level at bridge piers. The proposed technique is based on an array of Bragg grating temperature sensors, heated by an electrical circuit. The Bragg gratings in water sense a lower temperature than those buried in the river bed, because of the different heat scattering principles in the two situations. Furthermore the response of each sensor is slower if it is buried in the bed, with respect to the case it is in water. The paper presents laboratory tests, showing the method effectiveness and reliability, and it explains the advantages with respect to other more traditional methodologies to measure scour level.
\end{abstract}

\section{Introduction}

Bridge pier scour [1] is one of the most common causes of bridge collapses. The most critical situation is the flood condition, when pier scour depth can become high and its time-evolution is relatively fast. As available models are unable to predict scour development with sufficient accuracy, several methods have been developed allowing for in situ scour measurements [2-6]. Here they are not described one by one as it would result too long but many information can be found in the quoted references.

Among the most common measurement devices there are echo sounders, sounding rods and buried rods. All these methods present different drawbacks. For example echo sounders do not guarantee satisfactory measurement accuracy during floods, which are the most critical events for pier scour. This has pulled towards looking for new techniques able to overcome the mentioned drawbacks.

The main goal has been to find out a method providing reliable measurements under both standard and flood conditions. Furthermore, instrumentation safety has been considered essential. Once again this problem is mainly related to floods, as many suspended bodies can damage the measurement devices under this condition. Moreover other aspects have been accounted for, such as system costs and installation complexity.

The new considered approach [7] is based on fibre optics as this technology offers many advantages, if compared to the abovementioned traditional techniques. They are discussed in Section 3, after having introduced the method principles in Section 2. Then Section 4 presents some

\footnotetext{
a e-mail : stefano.manzoni@polimi.it
} 
laboratory tests performed to evaluate technique effectiveness and reliability. It will be shown that it possible to exploit two different principles to recognise scour level, increasing method reliability.

\section{Measurement method}

The basic idea is to adopt an array of temperature sensors based on fibre optics technology. These temperature sensors are Bragg gratings [8,9]. The array is set up in vertical direction, just close to the pier. Figure 1 presents the system layout where many different sensors are shown.

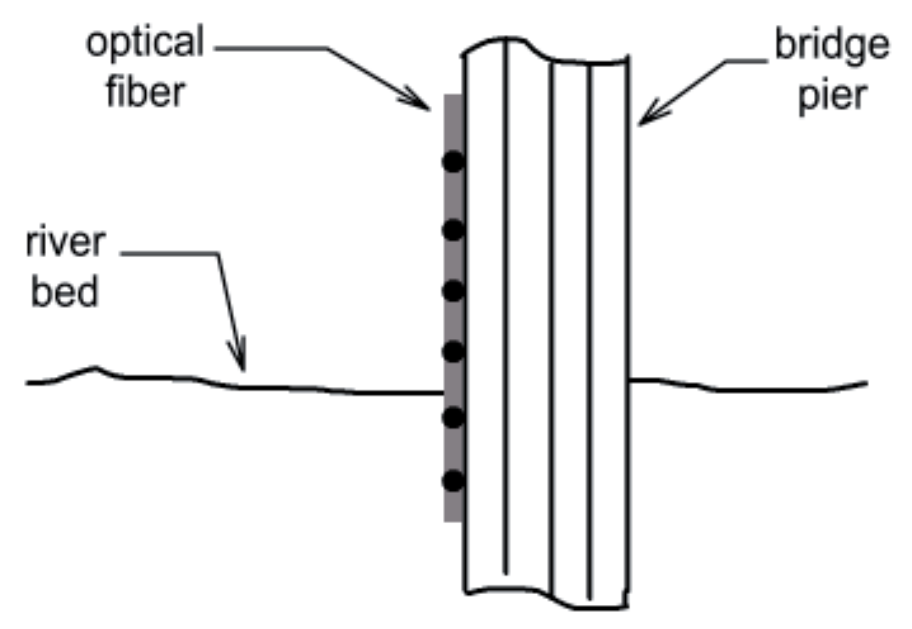

the black circles indicate the Bragg gratings

Fig. 1. Measurement system layout.

The fibre Bragg gratings (FBG in the following) are a sort of light filters. A broad band light is emitted into the fibre and reaches the FBGs. Each FBG reflects a part of this light. The reflected light has a very narrow spectrum centred on a certain wavelength (Bragg wavelength in the following), while the other part of light passes through the FBG.

When the FBG is interested by a strain due to a mechanical or thermal action or by a temperature change, the Bragg wavelength changes too. Thus the Bragg wavelength, measurable by means of an optical interrogator, can be considered as the output of a strain or temperature transducer. This means that different FBGs with well separated Bragg wavelengths can be inserted into the same optical fibre. The only requirement is that the Bragg wavelength value at the temperature or strain full-scale does not cross other sensor Bragg wavelengths. This fact has important fallouts in terms of both system simplicity and economical benefits. These points are discussed in Section 3. A further aspect is worth being mentioned. Here the interesting information is temperature, while strain is considered as a disturbance variable. It is therefore needed to prevent strain Bragg wavelength shifts. There are different ways to reach such a goal. In this case an uncoupling between thermal and strain effects has been obtained putting the whole fibre into a stainless tube structure (with an external diameter of $3 \mathrm{~mm}$ ) and gluing the fibre to the structure just in a single point, far away from the Bragg gratings. This allows the fibre not to be affected by strains acting on the outer structure.

The fibre containing the various FBGs is heated by an electrical circuit thanks to the dissipation Joule effect. The heat produced by the Joule effect is then scattered due to conduction and 
convection and the basic concept is that the temperature sensors in water should sense a lower temperature than those buried in the bed as the heat dispersion is much higher for the former ones. The principle is similar to that of a hot-wire probe [10] working with constant current. Thus it is expected that the different temperatures felt by the two sensor families can be a reliable tool for detecting the interface between flowing water and saturated soil forming the river bed. The knowledge of the height of each temperature transducer and its sensed temperature allows to know which is the scour level and the measurement resolution depends on sensor distance. A further approach can be adopted as well and it relies on the fact that, when the electrical circuit is switched on, the Bragg gratings in the bed have a slower response than those in water, in terms of sensed temperature. This is again due to the different heat scattering dominating phenomena for sensors in water and buried in the bed. Therefore the response velocity is a further parameter usable to estimate scour level.

The next section discusses the advantages provided by the new proposed methods, paying special care to the improvements with the already available scour measurement techniques.

\section{Technique advantages}

The new considered approach has different advantages with respect to more traditional measurement techniques. The main ones are mentioned here.

The first advantage is that every fibre can be equipped with many sensing elements. A single interrogation channel can therefore be used for some tens of sensors. On the other hand, when traditional temperature sensors are adopted, one acquisition channel must be dedicated to each transducer. The use of optical fibres thus gives a great advantage when the number of temperature sensors becomes high, in terms of both system complexity and cost. As the scour level resolution depends upon the distance (and thus on the number) of temperature sensors, an accurate measurement requires many sensors. In this case the proposed solution brings to the mentioned improvement.

Furthermore, the optical fibre principle is based on light and optics, while traditional sensors have electronics on board, so weak flowing currents and voltages. In this case the environment is the water, which is very harsh for electrical transducers. On the other hand, optical fibre performances are only lowly affected by the presence of water, giving a stable output. Therefore the proposed measurement system offers a much higher reliability and robustness, if compared to more traditional systems.

Finally, a further advantage is that the fibre can be easily protected against suspended body hits, thus having a good mechanical reliability.

The new proposed device is able to work properly in flood conditions, where many traditional approaches to measure scour level fail. This is an evaluable improvement as scouring is very critical under flood conditions because scour level can be very high and therefore critical for bridge failures.

\section{Experimental tests}

The measurement method has been investigated through an extensive laboratory activity. The tests have been carried out in a water channel with a section of $100 \mathrm{~cm}$ (base) $\times 70 \mathrm{~cm}$ (height).

A pier model has been placed inside the channel. The model was cylindrically shaped with an outer diameter of $16 \mathrm{~cm}$ of and a height of $80 \mathrm{~cm}$ (the water level was $60 \mathrm{~cm}$ ). The fibre was attached to the pier. This layout allows to test temperature sensors immersed in flowing water.

Nevertheless, it was also needed to simulate situations in which the temperature sensors are buried in the river bed. Another structure has been built to this purpose. It is a cylinder with an outer diameter of $30 \mathrm{~cm}$, thus greater than the external diameter of the pier model. This second additional cylinder could be placed around the pier model and the space between them could be filled up with 
debris, of the same kind as those usually present in river beds. These debris were maintained wet by means of a water jet.

The described layout allows to reproduce conditions similar to those of a river bed, very close to the real situation. Air and water temperatures were monitored by means of thermocouples.

The fibre was provided with three FBG temperature sensors and their spacing was $200 \mathrm{~mm}$. The sensitivity was nearly $0.01 \mathrm{~nm} / \mathrm{K}$ for all the sensors.

Concerning the heating system, three wires have been fixed along the stainless tube (Figure 2a) mentioned in Section 2. The total resistance of each wire of the electric circuit was about $50 \Omega$ and the voltage source they were linked to could be switched among six values $(5,10,15,20,25$ and $29 \mathrm{~V})$. The total length of each wire was about $2 \mathrm{~m}(1 \mathrm{~m}$ from the power supply down to the fiber bottom and $1 \mathrm{~m}$ from the fiber bottom up to the power supply as explained in Figure 2b). Table 1 gives the Joule effect dissipated power per meter of fibre for the six possible voltage supply values.
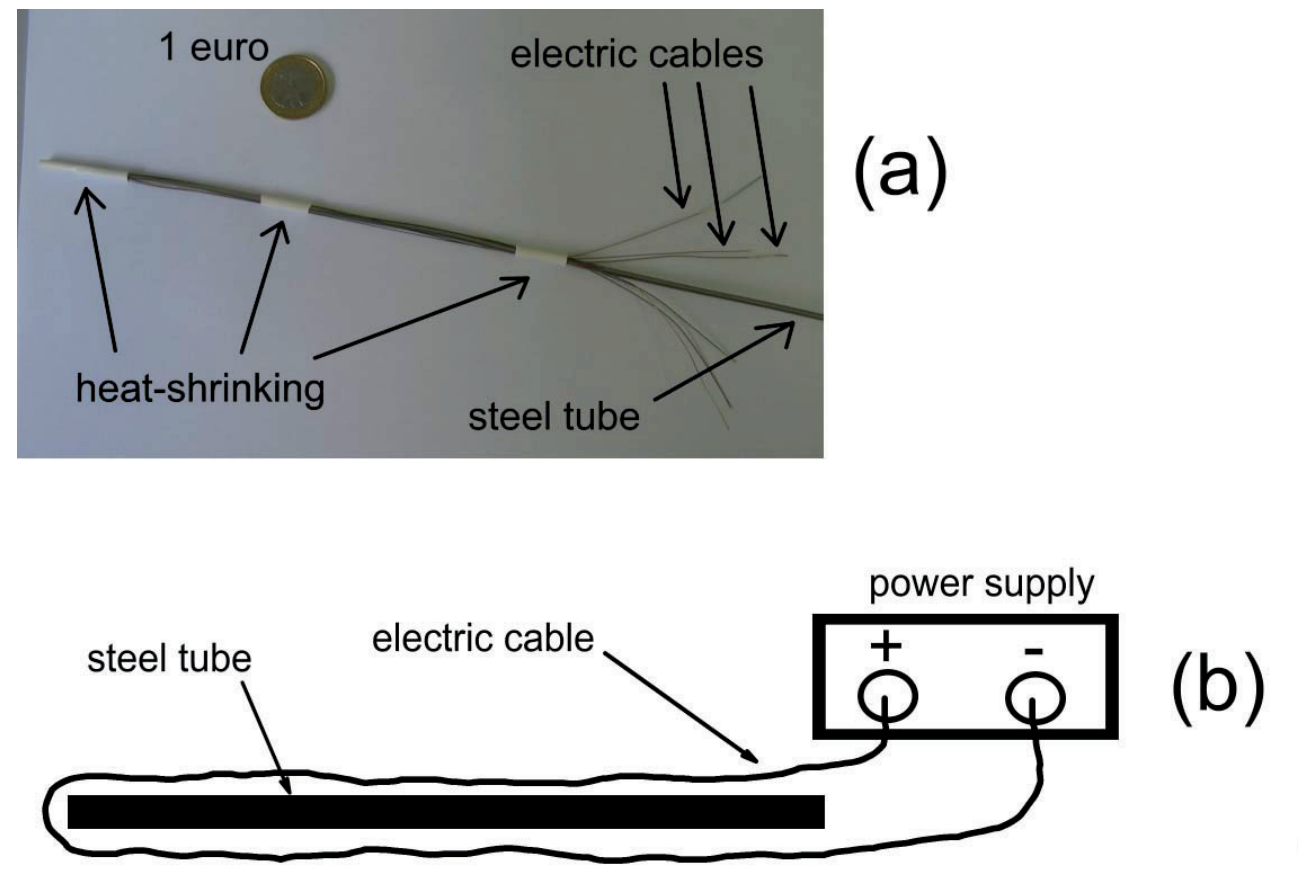

Fig. 2. Electrical wire layout.

Some experimental evidences have to be explained before passing to the test overview. Figure 3 shows the response of the same sensor in water (flow speed $=0.4 \mathrm{~m} / \mathrm{s}$ ) and in the debris, when the electric circuit is switched on (the dissipated power when the circuit was switched on was $50.5 \mathrm{~W} / \mathrm{m}$ in the shown case).

The experimental tests show that the steady-state temperature is different if the sensor is in water or buried in debris, as expected from theory.

The experimental sensor output time-histories are well approximated by the following law:

$$
T=\Delta T \bullet\left(1-e^{-\frac{t}{\tau}}\right)+T S
$$


where $T$ is the current output temperature, $\Delta T$ is the difference (given by the sensor itself) between the steady-state temperature and the starting temperature, $T S$ is the starting temperature and $\tau$ is a time constant.

Table 1. Tested electrical parameter values.

\begin{tabular}{|c|c|c|}
\hline Supply voltage [V] & $\begin{array}{l}\text { Number of } \\
\text { used wires }\end{array}$ & Dissipated power [Watt per meter of fibre] \\
\hline 5 & 1 & 0.5 \\
\hline 5 & 2 & 1.0 \\
\hline 5 & 3 & 1.5 \\
\hline 10 & 1 & 2.0 \\
\hline 10 & 2 & 4.0 \\
\hline 10 & 3 & 6.0 \\
\hline 15 & 1 & 4.5 \\
\hline 15 & 2 & 9.0 \\
\hline 15 & 3 & 13.5 \\
\hline 20 & 1 & 8.0 \\
\hline 20 & 2 & 16.0 \\
\hline 20 & 3 & 24.0 \\
\hline 25 & 1 & 12.5 \\
\hline 25 & 2 & 25.0 \\
\hline 25 & 3 & 37.5 \\
\hline 29 & 1 & 16.8 \\
\hline 29 & 2 & 33.6 \\
\hline 29 & 3 & 50.5 \\
\hline
\end{tabular}

The time constant $\tau$ of the two responses of Figure 3 is pretty different. $\tau$ in water is about $5 \mathrm{~s}$ and in the debris is about $11 \mathrm{~s}$. This is a further measurement strategy variable which could be adopted to understand if a sensor is immersed in water or in the river bed, and thus capable to measure scour level. This point is going to be discussed again in the following.

The main variable governing the system performances is the value of the dissipated power. Its effect has been investigated and the main results are provided here.

Figure 4 shows the $\Delta$ temperature (steady state temperature - starting temperature) as a function of the dissipated power in Watt per meter of fibre for one of the three sensors (that already considered in Figure 3), while Table 2 presents the data concerning the $\tau$ trend. As the $\tau$ value is almost constant when the dissipated power value changes, these results are summarised calculating the mean and the standard deviation on all the performed tests both for sensor in water and buried in debris. The considered sensor is that considered also in Figures 3 and 4. The results are similar for the two remaining FBGs. 
Figure 4 and Table 2 show that it is possible to recognise the sensor position (buried in the bed or in water) and thus the scour level relying on both the mentioned approaches, provided that the dissipated power per meter of fibre is not too low.

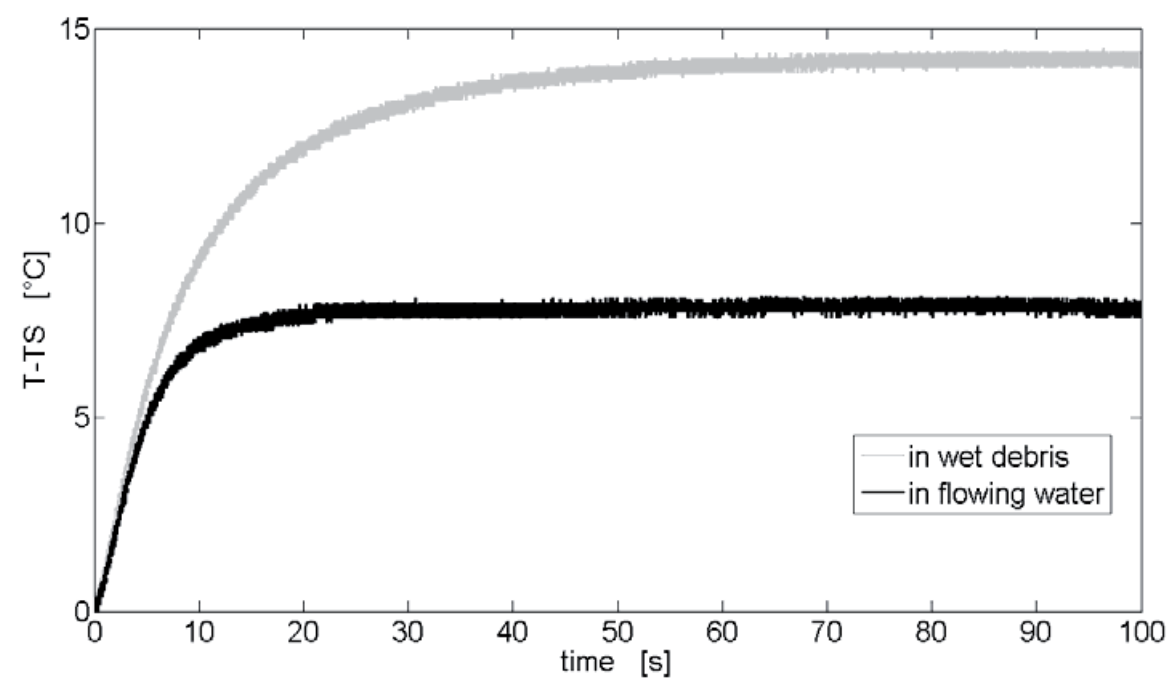

Fig. 3. $\Delta$ temperature $T-T s$ (Equation 1) time-histories for the same sensor in water and in debris when the electric circuit is switched on (sampling frequency $=1 \mathrm{kHz}$ ). The dissipated power per meter of fibre was $50.5 \mathrm{~W} / \mathrm{m}$.

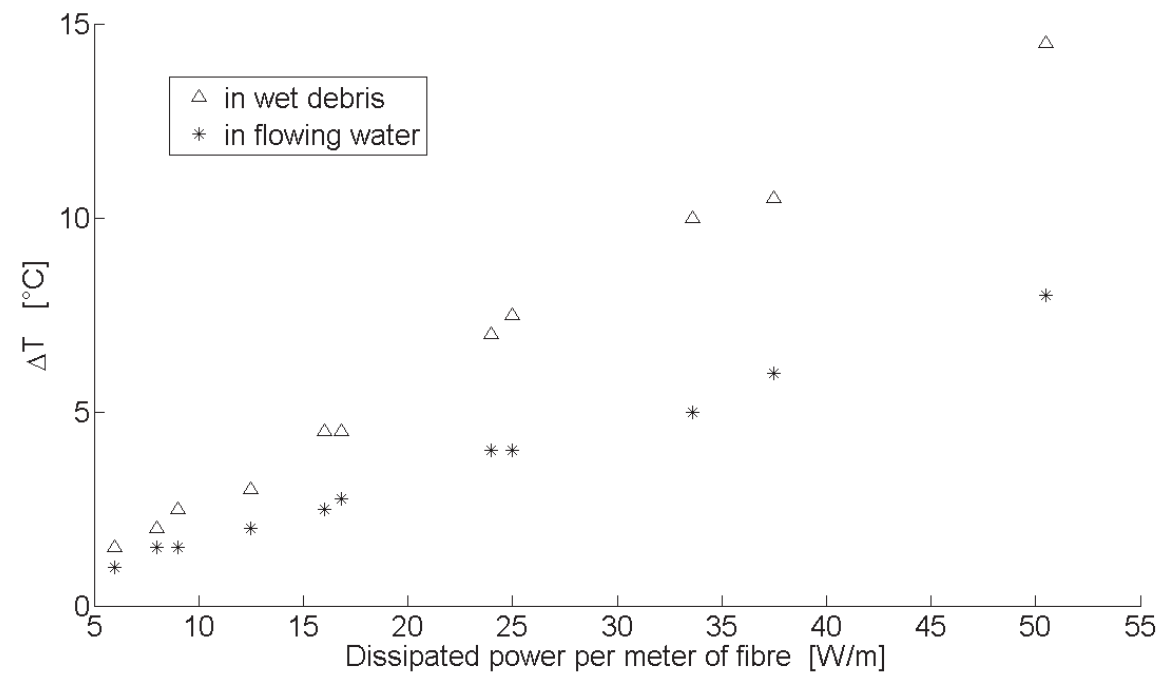

Fig. 4. $\Delta$ temperature (steady state temperature - starting temperature) ( $\Delta T$ in Equation 1$)$ as a function of the dissipated power in Watt per meter of fibre. The sensor is that already considered in Figure 3. 
Table 2. Mean and standard deviation values of $\tau$ for the sensor considered in Figures 3 and 4.

\begin{tabular}{|c|c|c|}
\hline Calculated parameter & Sensor in flowing water & Sensor in wet debris \\
\hline$\tau$ mean value $[\mathrm{s}]$ & 4.95 & 10.71 \\
\hline$\tau$ standard deviation value $[\mathrm{s}]$ & 0.69 & 1.74 \\
\hline
\end{tabular}

\section{Conclusion}

The paper has presented an innovative method to measure scour level at bridge piers. This is based on FBG temperature sensor arrays heated through an electric circuit dissipating heat. The main method advantage is that it is able to accurately give the scour level also in flood conditions, which are the most critical situations where many traditional techniques fail.

The new approach relies on two possible strategies as temperature sensors in water and debris are characterised by different outputs and by different time responses when the electric circuit is switched on. This double working principle, tested by means of laboratory experimental tests, increases method reliability.

\section{References}

1. F. Ballio, E. Orsi, Time evolution of scour around bridge abutments, Water Engineering Research 24 (2001) 243-259.

2. National Cooperative Highway Research Program (NCHRP), Report 396 - Instrumentation for measuring scour at bridge piers and abutments, Transportation Board and National Research Council (1997).

3. F. De Falco, R. Mele, The monitoring of bridges for scour by sonar and sedimetri, Independent Nondestructive Testing and Evaluation International 352 (2002), 117-123.

4. S. Radice, F. Ballio, A non-touch sensor for local scour measurements, Journal of hydraulic research 411 (2003), 105-108.

5. Y-B. Lin, J-C. Chen, K-C. Chang, J-C. Chern, J-S. Lai, Real-time monitoring of local scour by using fiber Bragg grating sensors, Smart Materials and Structures 14 (2005), 664-670.

6. K-F. Lo, S-H. Ni, Y-H. Huang, X-M. Zhou, Measurement of unknown bridge foundation depth by parallel seismic method, Experimental Techniques 331 (2009) 23-27.

7. A. Cigada, F. Ballio, F. Inzoli, Hydraulic Monitoring Unit, application for international patent $\mathrm{n}$. PCT/EP2008/059075, publication n. WO/2009/013151 (2009).

8. K.O. Hill, G. Meltz, Fiber Bragg grating technology fundamentals and overview. Journal of Lightwave Technology 158 (1997), 1263-1276.

9. S.W. James, M.L. Dockney, R.P. Tatam, Simultaneous independent temperature and strain measurement using in-fibre Bragg grating sensors. Electronics Letters 3212 (1996), 1133-1134.

10. E.O. Doebelin, Measurement systems: application and design, Fifth Edition (The McGraw-Hill Companies, 2004). 